To appear in "Magnetic Coupling between the Interior and the Atmosphere of the Sun", eds. S. S. Hasan and R. J. Rutten, Astrophysics and Space Science Proceedings, Springer-Verlag, Heidelberg, Berlin, 2009.

\title{
Angular-Momentum Coupling through the Tachocline
}

\author{
D. O. Gough ${ }^{1,2}$ \\ 1 Institute of Astronomy, University of Cambridge, UK \\ 2 Department of Applied Mathematics and Theoretical Physics, University of \\ Cambridge, UK
}

\begin{abstract}
Summary. Astronomical observation of stellar rotation suggests that at least the surface layers of the Sun have lost a substantial amount of the angular momentum that they possessed at the beginning of the main-sequence phase of evolution; and solar-wind observations indicate that magnetic coupling is still draining angular momentum from the Sun today. In addition, helioseismological analysis has shown that the specific angular momentum at the top of the almost uniformly rotating radiative interior is approximately (although not exactly) the same as the spherically averaged value at the base of the (differentially rotating) convection zone, suggesting that angular momentum is being transported through the tachocline. The mechanism by which that transport is taking place is not understood. Nor is there a consensus of opinion. I review some of the suggestions that have been put forward, biassing my discussion, no doubt, according to my own opinions.
\end{abstract}

\section{Introduction}

First, I recall that the Sun must have started life with much more angular momentum than it has today. There is considerable circumstantial evidence to support that view, the strongest, in my opinion being that stars are formed from interstellar gas clouds whose typical intrinsic angular momentum per solar mass is some $10^{7}$ times that of the Sun today.

Almost all of the initial angular momenta of almost all stars was lost before the gas was even able to condense to stellar proportions. It is likely that during the later stages of gravitational collapse a circumstellar disc is formed which removes angular momentum from the star by magnetic coupling. The process by which that occurs is a very important arena of research, and a great deal of very interesting work is being done to understand it. But that is beyond the scope of this discussion.

What I might point out, however, is that angular-momentum loss appears to continue after the star has been established and most of the disc has departed, as is evinced by the observed decline with age in the rotation rates of the surfaces of stars in young clusters. It should also be acknowledged that 
even today the Sun is losing angular momentum, via magnetic coupling to the solar wind, although at a rate that is rather less than that suggested by the rotation rates obtained by a simple extrapolation to the solar age of apparent angular-momentum loss rates from young-cluster stars.

The "simple" extrapolation is based on the assumption that young stars rotate almost uniformly, as does the Sun today, which may not be realistic. It may be that the mechanical coupling between the surface layers and the deep interior is insufficient to transfer angular momentum fast enough to render the surface rotation rates of young stars direct indicators of the rotation of their interiors. That would explain the initial rapid slow-down, but it would also imply that the "initial" angular momentum of the main-sequence Sun was lower than average.

Was there a tachocline in those early main-sequence days? And if so, was its structure similar to that of today? I suggest that there was a tachocline, and that its structure was similar, although in the early days the shear was probably stronger.

A second, very important, matter that must be considered is whether the early Sun, and, of course, other similar young stars, harboured substantial large-scale magnetic fields throughout their radiative interiors; and by "substantial" I mean one that is intense enough to transport angular momentum on a timescale not significantly greater that the age of the star. I invite you to entertain the possibility, partly because I regard it as being most unlikely that the Sun had lost all the field that had pervaded the interstellar gas from which it had condensed. Indeed, I believe that there remains a dynamically important residual even today, simply because there appears to be no cogent argument for maintaining the observed uniform rotation of the radiative interior by any non-magnetic process. I must hastily point out that this opinion is not universally accepted, so I shall return to it later.

Before proceeding, it is useful to state the principal properties of the Sun that must not be ignored: The Sun is basically in hydrostatic balance; approximately the outer 30 per cent by radius ( $65 \%$ by volume, $2 \%$ by mass), supporting 15 per cent of the moment of inertia, is in a state of turbulent convection. The rest is relatively quiescent. There, energy that has been generated in the nuclear-reacting core is transported by radiative diffusion. The stratification of both the radiative interior and most of the convection zone has been well established by helioseismology, as also has the angular velocity, $\Omega$, which I now describe.

In the bulk of the convection zone $\Omega$ increases with co-latitude, from about $2.3 \times 10^{-6} \mathrm{~s}^{-1}$ (corresponding to a period of about 31 days) at the poles (where it cannot be measured accurately) to about $2.86 \times 10^{-6} \mathrm{~s}^{-1}$ (corresponding to a period of about 25.4 days) at the equator; it is roughly independent of radius, although, as noted by Gilman \& Howe (2003), the $\Omega$ contours are more nearly inclined by a constant $27^{\circ}$ from the axis. The radiative interior (at least outside the energy-generating core in which reliable measurements have not yet been made) rotates approximately uniformly, with angular velocity 


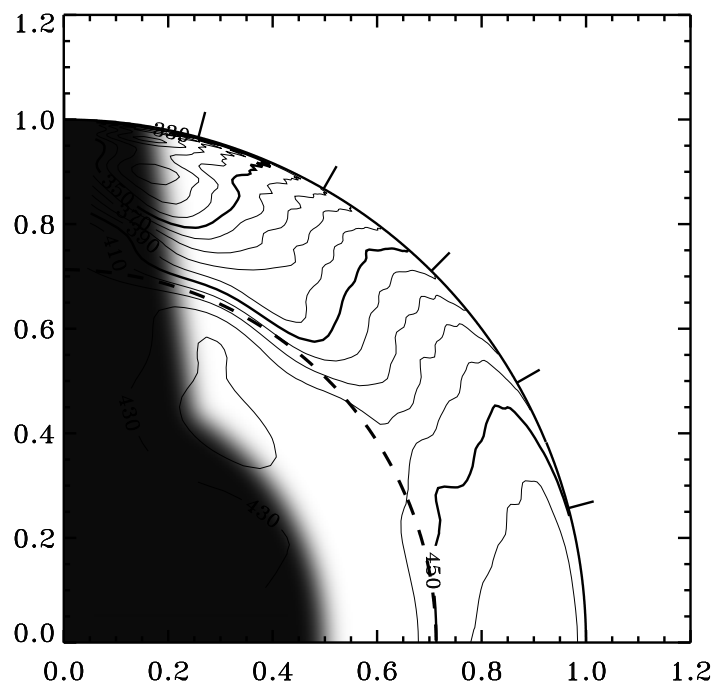

Fig. 1. Contours of constant rotation rate $(\Omega / 2 \pi)$ in a quadrant of the Sun as inferred by Schou et al. (1998)). The contours, two of which are labelled, are separated by $10 \mathrm{nHz}$. The continuous quarter-circle represents the photosphere, the dashed quarter-circle the base of the adiabatically stratified convection zone. The tachocline is clearly visible, the shear layer extending outwards from the radiative interior into the convection zone, the angular velocity increasing outwards near the equator and decreasing near the poles; the vertical shear vanishes at a latitude of about $30^{\circ}$. The darkened region is where reliable helioseismological inferences could not be drawn.

$\Omega_{0} \simeq 2.67 \times 10^{-6} \mathrm{~s}^{-1}$, which is 0.93 of the equatorial angular velocity in the convection zone (see Fig. 1). The radiative and convective regions are separated by a thin shear layer, known as the tachocline, which is too thin to be reliably resolved directly by seismology (or any other means); indirect estimates range between 2 per cent and about 8 per cent of the solar radius, with some indication that the tachocline is thicker near the poles than near the equator. I should point out that the variation in the estimates is due partly to differences in the way the tachocline is perceived, a matter on which I shall expand later. The base of the convection zone is likely on theoretical grounds to be fairly accurately spherical.

\section{Some initial remarks on tachocline dynamics}

My brief for this presentation is to discuss how angular momentum is transported from the radiative interior to the convection zone through the tachocline. It is not unreasonable to suppose that the transport has always been outwards, at least since the Sun arrived on the main sequence. I say this 
partly because the Sun is, and presumable always has been, losing angular momentum through the photosphere to the solar wind via magnetic stresses, and partly because most of the Sun has been expanding slightly, and preferentially in the convection zone, during its main-sequence evolution (the core has contracted). It is, of course, of interest to understand how the radiative interior has slowed down through this process to its current, uniformly rotating, state, which raises the question of how angular momentum is redistributed to maintain the uniformity of rotation. Here I shall maintain the stance that the rotation is uniform because on timescales of relevance the interior is rigid, held so by a large-scale primordial magnetic field. I shall mention points of view to the contrary at the end of the presentation.

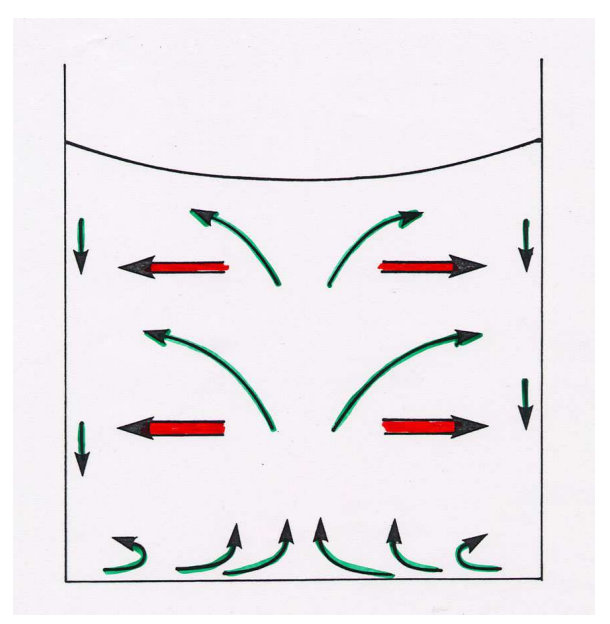

Fig. 2. Cartoon of a stirred cup of tea (with no handle). The double arrows represent the centrifugal force excerted on the fluid, which is resisted by the sides of the cup inducing a radial (i.e., pointing away from the axis) pressure gradient which causes the surface of the tea to be concave. The centrifugal force is much weaker near the bottom of the teacup where viscous stresses inhibit the rotation, leaving a ("statically") unbalanced pressure gradient to drive an inward flow in a thin viscous (Ekman) boundary layer, denoted by the upwardly curving single arrows. The return flow completing the circulation is also indicated by single arrows.

I now discuss a fundamental dynamical phenomenon which occurs universally in rotating shearing fluids, namely that associated with the differential rotation is a meridional circulation. This phenomenon was discussed by Einstein (1926) to explain the meanders of gently flowing rivers, and it is responsible for the rapid spin-down of a stirred cup of tea. Consider the fluid in the cup illustrated in Fig. 2. The fluid is rotating, slowly, about a vertical axis, and the centrifugal force in the body of the fluid, denoted by the double arrows, is resisted by the impermeable wall of the cup, augmenting the pressure to produce an outwardly directed component to the pressure gradient. The higher pressure farther from the axis supports a greater head of fluid (which is essentially in vertical hydrostatic balance under gravity), whose upper surface is therefore concave. Very near the bottom of the cup, however, the rotation of the fluid is impeded by viscous stresses against the rigid base; the centrifugal force is unable to balance the outward component 
of the pressure gradient (which evidently remains unchanged in view of the vertical hydrostatic balance), the residual of which drives an inward flow (denoted by the single, curved, arrows), drawing in fluid from the side of the cup to replace it. The inward flow is deflected upwards into the body of the fluid, where it expands away from the axis, and subsequently descends near the wall of the cup to complete the circulation. If one were to view the fluid from above, concentrating on a portion of an outer annulus, one can liken it to a bend in a river: the locally rotating stream produced by the bend causes an inward flow near the bottom of the river which erodes mud, sand and stones, transporting them from the outer bank to the inner bank and thereby accentuating the bend, resulting finally in a meander.

The inward flow near the base of the teacup occurs in a thin boundary layer, of thickness $\delta$, say, whose basic structure had been analysed by Vagn Ekman (and has subsequently been named after him) in his doctoral thesis in 1902 for the purpose of explaining why Arctic ice does not drift in the same direction as the prevailing wind. It explains also why a stirred cup of tea slows down faster than the characteristic viscous diffusion time $\tau_{\mathrm{d}}=R^{2} / \nu$, where $R$ is the radius of the teacup and $\nu$ is the viscous diffusion coefficient: the angular momentum in the tea is transferred to the cup in the boundary layer on the shorter timescale $\delta^{2} / \nu$, and the angular velocity in the essentially inviscid flow elsewhere declines because the angular-momentum-conserving fluid is drifting away from the axis of rotation (it can be shown that the flow near the outer wall occurs in a layer considerably thicker than $\delta$, so angularmomentum transfer there can be ignored). When all this is put together, the final outcome, as in so many other cases of fluid flow with thin diffusive (boundary) layers, is that global evolution occurs on a timescale which is the geometric mean of the characteristic dynamical and the large-scale diffusive times. In the case of a cup of tea, $\tau_{\mathrm{d}} \simeq 1 / 2$ hour, and if one has stirred at a rate of two per second, the dynamical time is $1 / 2$ second; therefore the tea slows down in about $1 / 2$ minute. Note that that spin-down time is also the characteristic circulation timescale.

The solar tachocline is broadly similar. The rigid radiative interior, which is very strongly stably stratified (the characteristic buoyancy restoring timescale is about an hour - the characteristic period of grave g modes - which is very much shorter than even the rotation period), acts like the rigid impermeable base of the teacup, above which is fluid which is caused to rotate differentially by the anisotropic Reynolds stresses, and associated Maxwell stresses, in the convection zone. Even the simplest dynamical description of the boundary layer (tachocline) that separates the two regions is considerably more complicated than an Ekman boundary layer (Gough \& McIntyre 1998); the basic dynamics of the meridional circulation in the convection zone is also somewhat different, being maintained by "gyroscopic pumping" by the anisotropic turbulent stresses rather than a spinning down like a cup of tea that is no longer being stirred. Near the equator, where the fluid in the convection zone is rotating faster than the "rigid" interior, there is a (poleward) essentially 


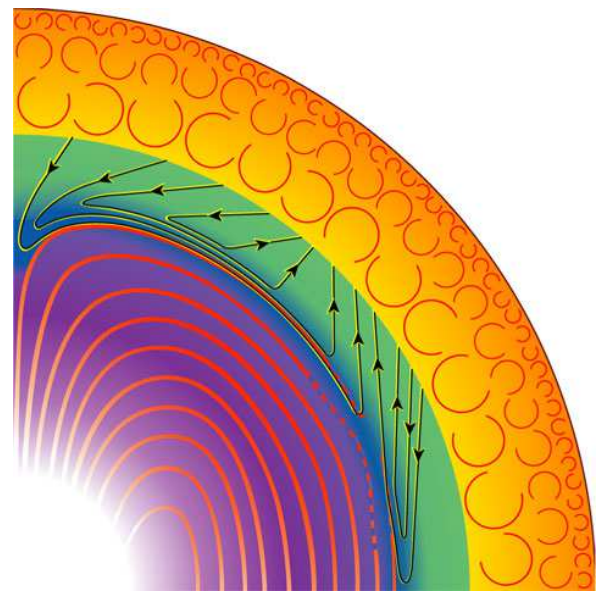

Fig. 3. Quadrant of the Sun indicating the gyroscopically driven meridional flow (lines with arrows) in the tachocline (from Gough \& McIntvre 1998). The width of the tachocline has been artificially exaggerated to render the flow visible. Circular arcs represent convective eddies, and the broad lines beneath the tachocline represent lines of magnetic field - they are dashed near the upwelling region, where their geometry is even more uncertain than elsewhere and where they are probably drawn into the convection zone, providing a source of Maxwell stress that may locally quench the shear.

horizontal flow in the tachocline towards the axis of rotation; contrarily, near the poles, where the convection zone rotates more slowly than the interior, the tachocline flow is equatorward. In both regions the motion draws fluid in from the convection zone (or, if one prefers, is pushed aside by the fluid descending from the convection zone from whence it has been pumped by the rotating anisotropic turbulent stresses). The opposing flows in the tachocline meet somewhere at mid latitudes, and, being unable to penetrate significantly into the radiative zone below, they are forced to rise back into the zone above, as in a cup of tea. The flow is illustrated in Fig. 3, where lines of interior magnetic field are also depicted.

\section{The early history of dynamical tachocline models}

The existence of the tachocline was first recognized theoretically by Spiegel (1972) in his study of the spin-down of the Sun. It attracted little attention at the time, and was hardly discussed until the existence of the tachocline was established seismologically (Brown \& Morrow 1987; Christensen-Dalsgaard \& Schou 1988; Brown et al. 1989; Dziembowski et al. 1989). A prime motivation for the early consequent studies was to explain how the radiative interior of the Sun could rotate uniformly in the face of the non-uniform stresses imposed by the differentially rotating convection zone, although many authors make a different emphasis, addressing why the transition between the differentially rotating convection zone and a putative uniformly rotating interior is so thin. The conclusion is that a thin tachocline is indeed plausible, perhaps even inevitable.

The first dynamical discussion of the matter was provided by Spiegel \& Zahn (1992), who proposed that turbulence in the stably stratified tachocline was essentially layerwise two-dimensional, having negligible vertical motion and 
being horizontally isotropic so that it acted like a powerful (constant) viscosity on horizontal spheres. The turbulence appeared to be generated by the latitudal rotational shear itself, and was presumed to be sufficiently intense actually to quench at depth the shear that produces it. Subject to those assumptions a tachocline was elegantly constructed so as to reproduce the helioseismological findings.

One of the obvious deficiencies of the Spiegel-Zahn model is that it predicts the wrong angular velocity $\Omega_{0}$ for the uniformly rotating core: $0.90 \Omega_{\mathrm{eq}}$ rather than the observed value of $0.93 \Omega_{\mathrm{eq}}$, where $\Omega_{\mathrm{eq}}$ is the equatorial angular velocity in the convection zone. This quantity had already been discussed by Gough (1985), who pointed out that its value would have been $0.96 \Omega_{\text {eq }}$ had the local shear stress-rate-of-strain relation at the base of the convection been independent of latitude, as would be the case with a thin tachocline exerting a shear stress produced by a uniform isotropic viscosity and (artificially) supporting no meridional flow. It is an important diagnostic of tachocline models, partly because it is an overall measure of the differential angular-momentum transport and partly because its latitudinal average can be measured quite precisely by seismology. Indeed, in those days it appeared that the spherically averaged angular velocity in the radiative interior might drop below even $0.90 \Omega_{\text {eq }}$ (Duvall et al. 1984; Gough 1984), which might actually be the case in the core (Elsworth et al. 1995; Basu et al. 1997). The reduction of $\Omega_{0}$ in the Spiegel-Zahn model appears to result principally from the latitudinal transport of angular momentum by the shear-turbulent viscous stresses in the tachocline acting upon the meridional circulation, which behave differently from the Reynolds stresses in the convection zone resulting from three-dimensional turbulence driven by buoyancy.

Spiegel \& Zahn's assumptions were challenged some time later Gough \& McIntyre 1998), principally because rotating layerwise two-dimensional turbulence in nature does not act in such a way as to quench shear. Nor, in general, does any other known purely fluid-dynamical (non-magnetic) motion, such as the dissipation of gravity waves generated near the base of the convection zone, which Kumar \& Quataert (1997) and Zahn et al. (1997) had erroneously (e.g., Gough 1997) argued. It was therefore concluded that only if the radiative interior were held rigid by a sufficiently intense magnetic field could its angular velocity be uniform. The flow descending into the tachocline at low and high latitudes (see Fig. 3) advects the outwardly diffusing magnetic field to maintain a field-confining balance which plays a role in determining the thickness of the tachocline. At mid latitudes, where the rotational shear is small, the ascending return flow is likely to drag field into the convection zone. There the dynamics is difficult to analyse, which is why the field lines in Fig. 3 have been drawn dashed. In their admittedly grossly oversimplified model, Gough \& McIntyre assumed the field to be basically dipolar (a reasonable assumption, because the dipole component of a complicated primordial field decays the most slowly), purely poloidal (not so reasonable because such fields are not stable (Markey \& Tayler 1973); a numerical simulation of field decay in 
an idealized star by Braithwaite \& Spruit (2004) resulted in a dipole field with a toroidal component), and with, for simplicity, its axis aligned with the axis of rotation. Although those assumptions are bound not to be satisfied exactly, it is unlikely that any plausible deviation from them would invalidate the basic underlying arguments. In both the Spiegel-Zahn and the Gough-McIntyre models, angular momentum is advected through the bulk of the tachocline by the meridional circulation. It appears to be the case that the overall strength of that flow is insensitive to the details of the tachocline boundary-layer structure, as is perhaps the case also of the cup of tea (recall that the time to spin down a cup of tea is the geometric mean of two global times that take no explicit account of the Ekman boundary-layer structure); the ventilation timescale (quoted by Gough and McIntyre but not explicitly by Spiegel and Zahn) of the meridional circulation in the solar tachocline is of order $10^{6} \mathrm{y}$.

It has often been mentioned that Rüdiger \& Kitchatinov (1997) had already pointed out that the radiative interior could be held rigid by a horizontal magnetic field of sufficient intensity, and that the shearing interface could be thin if the vertical variation of the field were somehow maintained. However, no cogent dynamical argument addressing how the field could be confined was offered. Instead, it was merely stated that the field would not diffuse into the convection zone because the (eddy) diffusivity there is so high; no explicit account was taken of the dynamical implications of the necessarily consequent high diffusion rate.

In the model by Gough \& McIntyre, an additional angular-momentum conduit is provided by the magnetic field that is presumed to penetrate the tachocline at mid latitudes. In the absence of instability (an unrealistic circumstance, but one to consider as a benchmark), any (imposed) vertical rotational shear would stretch the field into a gyre, creating an increasing opposing torque until diffusion quenches its growth. Perhaps that torque quenches the shear instead, leading to a tachocline with essentially no shear at all (with apologies for the oxymoron) in the region (in each hemisphere) of penetrating field. T. Sekii and I are currently seeking seismological evidence for such a shear-free region, which, according to current seismological inference (see Fig. 1), must be located near latitudes $\pm 30^{\circ}$.

The $10^{6}$-year tachocline ventilation time is very much shorter than the $10^{10}$-year evolution timescale of the Sun. Therefore, on that timescale the net transport of angular momentum across the tachocline essential vanishes, unless the Sun is undergoing a deeply seated torsional oscillation (for which there is actually some helioseismological evidence, provided by Howe et al. (2000), who report a 1.3-y oscillation in $\Omega$ near the equator immediately above and below the tachocline), in which case it is the time-averaged angular momentum transport that vanishes. That constraint, coupled with the (modeldependent) latitudinal variation of the angular-momentum flux, determines the equilibrium (average) angular velocity $\Omega_{0}$ of the radiative interior. 
Note that I have in mind a state of rotation which is essentially steady (with perhaps a gentle superposed oscillation) over the ventilation timescale; on the much longer timescale of the main-sequence evolution of the Sun, a minute shear would transport angular momentum enough to maintain the angular-velocity balance between the braking convection zone and the radiative interior.

I should point out that the confinement of the magnetic field by the generally downwelling meridional flow in the radiative interior, except in the presumably small upwelling zone at mid latitudes, is an essential feature of the dynamics proposed by Gough \& McIntyre. Without it, magnetic field would thread the tachocline into the convection zone where it would, in the mean, adopt (much of) the differential rotation of that zone and thereby exert a torque on the radiative region below, causing it to rotate differentially too. Some numerical simulations have provided examples of this general process (e.g., MacGregor \& Charbonneau 1999; Garaud 2002; Brun \& Zahn 2006).

An essential property of the dynamics, therefore, is that the downwelling velocity is strong enough to dominate the outward diffusion of the field, except of course at the very bottom of the tachocline where there is magnetic boundary layer - the magnetopause - of thickness $\delta$, which is much thinner than the thickness $\Delta$ of the essentially diffusion-free body of the tachocline. It goes without saying that in order to model the downwelling from the convection zone the fluid must be permitted to flow from the convection zone into the tachocline; any theoretical model separating the two regions by an artificial impermeable boundary is bound not to provide a faithful representation of the true dynamics.

\section{Some consequences of tachocline circulation}

It can hardly pass unnoticed that the latitude at which magnetic field might penetrate the tachocline is the same as that at which sunspots first appear at the start of a new cycle (see Fig. 4). Perhaps it is the radiative interior of the Sun that supplies the convection zone with a seed field with which to generate the magnetic cycle. I shall leave that remark undeveloped, one on which to ponder. Evidently magnetoydrodynamical processes, commonly called dynamo action in this context, process the field to produce its cyclic behaviour; but those processes may not sustain the field as in a true dynamo, instead declining with the decaying primordial field.

One process of which we can be sure is that the meridional circulation, driven by gyroscopic pumping in the convection zone, ventilates the tachocline and tends to mix any heavy elements that may have settled under gravity back into the convection zone. Since the ventilation time is very much less than the age of the Sun, that mixing is essentially complete in the Gough-McIntyre scenario; in the Spiegel-Zahn scenario the rapid horizontal diffusion opposes the vertical transport, augmenting the mixing time to a substantial fraction of 


\section{DAILY SUNSPOT AREA AVERAGED OVER INDIVIDUAL SOLAR ROTATIONS}
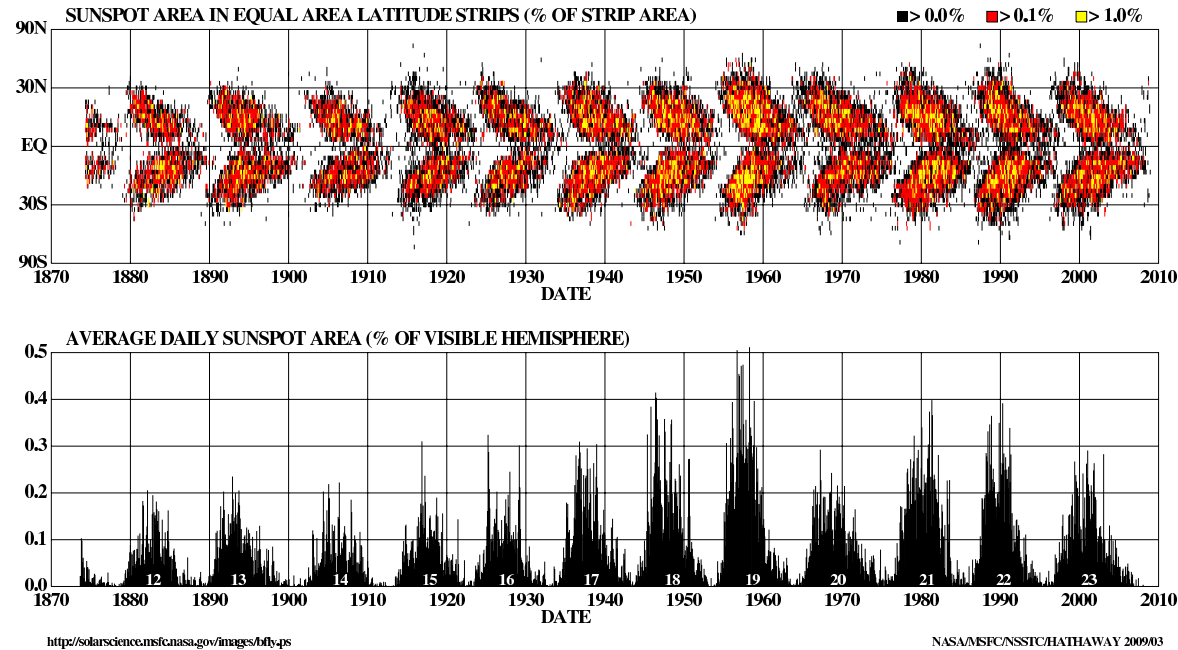

Fig. 4. Sunspot area (averaged over a solar rotation) plotted against time. The lower panel is a histogram of the total area; the upper panel indicates the latitudes of the spots (courtesy D. Hathaway, NASA Marshall Space Flight Center).

the solar age (Chaboyer \& Zahn 1992) and rendering it plausible that mixing is incomplete. In either case the outcome is a local reduction of the mean molecular mass of the tachocline material below that predicted by standard solar models, and a consequent augmentation of the sound speed, producing what has been called the tachocline anomaly (see Fig. 5). Calibration of this anomaly seismologically is the most precise way to determine the thickness of the tachocline because it uses the multiplet frequencies (mean over azimuthal order at given principal order and degree) of the seismic modes, rather than the less-well determined rotational splitting. However, the outcome depends on the premises upon which the theoretical reference solar model was built, and on the presumed evolution of the thickness of the tachocline. It may therefore not provide the most accurate determination.

It should be pointed out that the molecular-mass gradient (maybe a near discontinuity) created at the base of the tachocline is hydrostatically very stable: that is to say, that it cannot be significantly upset by the $10^{6}$-year meridional flow or the magnetic field that that flow confines (a basic point that was missed by Gough \& McIntyre when they suggested the possibility of a "polar pit" in the tachocline which might permit mixing of lithium to depths at which nuclear transmutations could occur today). Therefore the base of the tachocline is likely to be quite precisely spherical (actually, almost coincident with an equipotential surface).

It is appropriate here to point out that the tachocline calibration yields the thickness of only the layer in the radiative region that is homogenized 


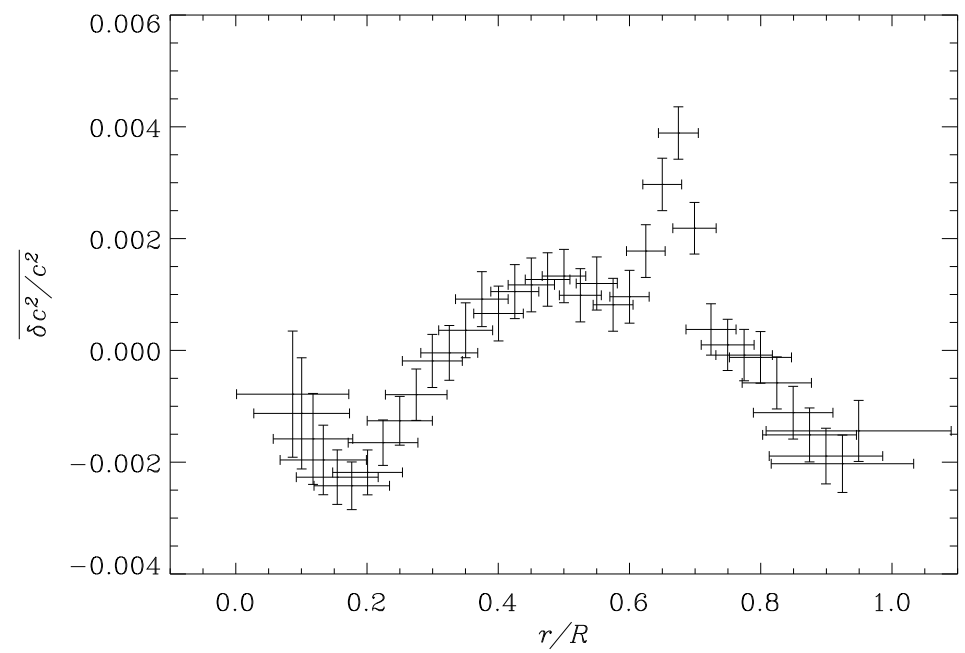

Fig. 5. Localized averages $\overline{\delta c^{2} / c^{2}}$ of the relative differences of the (spherically averaged) squares of the sound speeds in the Sun and in a standard theoretical solar model (Model S of Christensen-Dalsgaard et al. 1996), plotted against the location of the centres of the overaging kernels. The horizontal bars indicate the extent of the averaging (approximately full width at half maximum of the almost Gaussian averaging kernels), the vertical bars the standard errors (which are correlated with one another) arising from the formal uncertainties in the seismic frequency data, which are presumed to be independent of one another. The prominent sharp anomaly is in the region occupied by the tachocline beneath the convection zone, the base of which is situated at radius $r=0.713 R$, where here $R$ is the radius of the Sun (Christensen-Dalsgaard et al. 1991).

with the convection zone. That is precisely the layer which Spiegel and Zahn named the tachocline. Spiegel and Zahn, and Gough \& McIntyre after them, assumed for simplicity that the natural stresses in the convection zone are so strong that the tachocline does not react back on the angular velocity in the convection zone by an appreciable amount, so that essentially all the rotational shear resides in the radiative layer. Helioseismological evidence suggests that that is not exactly right (see Fig. 1), and that the shear has spread somewhat into the convection zone above, especially at high latitudes where vortex stretching is the greatest. There the dynamics is quite different; so it is perhaps prudent to reserve the term "tachocline" for only the radiative boundary layer, respecting Spiegel \& Zahn's precedent notwithstanding its etymological origins. A straightforward calibration of the sound-speed anomaly can then yield a tachocline thickness $\Delta$ of about $0.02 \mathrm{R}_{\odot}$, assuming complete mixing (Elliott \& Gough 1999). Incomplete mixing would yield a larger value. (At this point it is worth mentioning that complete mixing down to some level is not 
consistent with a recent seismological analysis by Christensen-Dalsgaard and Gough (unpublished), but nor is a simple incompletely mixed layer residing entirely beneath the adiabatically stratified convection zone.) Calibrations by fitting parametrized functions to the seismologically inferred shear layer near the base of the convection zone (e.g., Kosovichev 1996; Charbonneau et al. 1999; Corbard et al. 1999), however, depend, of course, on the fitting function adopted. They have yielded values of $\Delta$ ranging from about $0.03 \mathrm{R}_{\odot}$, (near the equator) to $0.08 \mathrm{R}_{\odot}$, (near the poles). Basu \& Antia (2003), for example, have reported that the surface defined by the mid-point of the transition is prolate; one can infer from their results, accepting the quoted uncertainties literally, that the base of the tachocline, defined by the shear, could perhaps be prolate too (with a pole-equator radius difference of about $0.01 \mathrm{R}_{\odot}$ ), but in the light of my earlier dynamical remarks on the matter I advise taking such a conclusion with a pinch of salt. There is some seismic evidence for solar-cycle variation of tachocline structure (e.g., Dziembowski \& Goode 1989; Baldner \& Basu 2008), but its nature is undetermined.

\section{The quest for a tachocline simulation}

There have been a series of attempts to address whether a magnetic-fieldconfining tachocline can actually be formed and maintained, using numerical simulations of different degrees of sophistication. Perhaps the most ambitious is a dynamical anelastic computation of Brun \& Zahn (2006), who modelled the outer half (by radius) of the radiative zone of a rotating Sun-like star harbouring a large-scale magnetic field. The inner and outer boundaries, at radii $0.35 R_{\odot}$ and $0.72 R_{\odot}$, were presumed to be impermeable but otherwise stress-free, maintaining a constant radial component of the entropy gradient; the magnetic field was matched smoothly onto potential fields outside the domain of computation. Of course, realistic diffusion coefficients could not be adopted; they had to be taken many orders of magnitude greater, but Brun and Zahn wisely maintained the correct ordering of their magnitudes, which they believed captured the essence of the real problem. They chose constant values, formally augmenting the thermal diffusivity $\kappa$ (in the tachocline) by a factor $6 \times 10^{5}$, the magnetic diffusivity $\eta$ by $2 \times 10^{8}$ and the viscosity by $3 \times 10^{8}$ (taking the diffusion coefficients in the tachocline to be those quoted by Gough 2007).

Brun \& Zahn carried out their computations from a variety of initial states, the most promising (for anyone interested in seeing a positive outcome, one might think) being one with a field well confined within the radiative interior. In all cases the field diffused through any semblance of a tachocline into the convection zone and took up its differential rotation, imparting it to the radiative interior beneath. In most of the simulations a potential magnetopause would have occupied the entire tachocline ( $\delta \simeq \Delta$, rather than $\delta \ll \Delta$ as found by Gough \& McIntyre). A simulation was run with $\delta \simeq \Delta / 3$; the differences 
in the result were barely noticeable. Brun and Zahn concluded that other processes must therefore be invoked to explain the tachocline structure, such as gravity-wave transport of the kind discussed by (Charbonnel \& Talon 2005; see also Charbonnel \& Talon 2007) or a rigidly generated oscillating dynamo field of the kind considered by Forgács-Dajka \& Petrovay (2001, 2002); I shall return to those matters later.

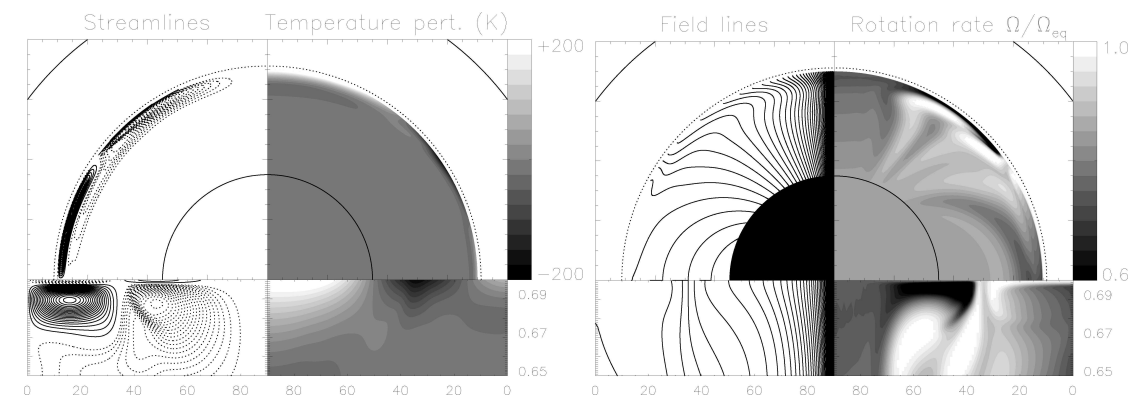

Fig. 6. Tachocline model by Garaud \& Garaud (2008), with imposed vertical velocity through the upper boundary as in Fig. 7. In the quadrants are indicated (on the left) streamlines (left) - solid for clockwise circulation, dashed for anticlockwise - and magnetic field lines (right), and (on the right) greyscale representations of temperature perturbations from the spherically symmetric background state (left) and the angular velocity $\Omega$ (right) measured in units of the equatorial angular velocity $\Omega_{\text {eq }}$ of the convection zone. Beneath each quadrant is an enlargement of the tachocline region, plotted against latitude and radius.

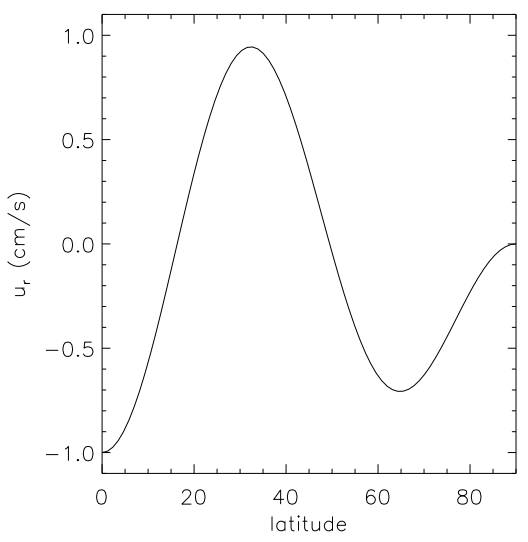

Fig. 7. Radial velocity component imposed by Garaud \& Garaud (2008) at the base of the putative convection zone, plotted as a function of latitude. 
A different approach has been taken by Garaud and her colleagues - the discussion by Garaud (2007) puts it into context - who carried out steadystate axisymmetric computations of a model of the radiative zone, the temporal and geometrical simplifications being made with the intention of rendering it possible to reduce the diffusion coefficients well below those truly attainable in resolved three-dimensional time-dependent simulations, and, it is hoped, to values even low enough for the interior magnetic field to be contained by the tachocline flow. She presumed the convection zone to be magnetically infinitely diffusive, and in her first paper (Garaud 2002) adopted an impermeable but otherwise stress-free boundary between the convection zone and the radiative interior, as did Brun \& Zahn (2006) after her. She too always found the magnetic field to penetrate the tachocline, particularly in the polar regions, and so impart a differential rotation on the radiative interior. But with her realization of the importance of the downwelling from the convection zone at low and high latitudes driven by the gyroscopic pumping Garaud \& Brummell 2008), and the development of a new numerical procedure, she succeeded in collaboration with her brother (Garaud \& Garaud 2008) to compute models of the outer part of the radiative zone $\left(0.35 \mathrm{R}_{\odot} \leqslant r \leqslant r_{\mathrm{c}}\right.$ with $r_{\mathrm{c}}=0.7 \mathrm{R}_{\odot}$ being the presumed base of the convection zone) in which fluid with yet lesser diffusion is made to descend from and ascend into the convection zone in a prescribed way.

Garaud \& Garaud (2008) demonstrated that if the upper boundary were presumed to be impermeable, the vertical flow could never be strong enough to attain a magnetic Reynolds number high enough to contain the field, no matter how low the diffusion coefficients might be, thereby explaining why all previous attempts to obtain a plausible numerical model of the tachocline had failed. When motion through the upper boundary was introduced, however, a dynamical balance between advection and diffusion of magnetic field qualitatively similar to that described by Gough \& McIntyre (1998), with angular momentum transported principally by advection in the upper part of the tachocline and by Maxwell stresses in the lower part, could in some situations be achieved, although the solutions also revealed a number of previously unrecognized subtelties. Moreover, the configuration adopted by the flow and the field is not unlike that found in the recent study by Wood \& McIntyre (2007) which extended the analysis of the Gough-McIntyre model to the vicinity of the poles.

As in all numerical studies to date it was necessary for Garaud and Garaud to augment the diffusivities by large factors, which they did in such a way as to maintain the hierarchy of characteristic boundary-layer thicknesses that might be expected to be encountered: the magnetic diffusivity $\eta$ was augmented by a factor $\tilde{f}$, and the kinematic viscosity $\nu$ and radiative thermal diffusivity $\kappa$ were adjusted accordingly to yield a Prandtl number $\nu / \kappa$ that is 10 times solar and a magnetic Prandtl number $\nu / \eta$ that is 0.1 solar. Figure 6 shows the solution for the least diffusive case that Garaud \& Garaud were able to compute with an upper permeable boundary, having $\tilde{f}=8 \times 10^{9}$. (It 
should be pointed out that this value is actually more than 10 times higher that what was formally employed by Brun \& Zahn (2006), but on the other hand the spatial resolution of the numerical scheme that was employed was more than 10 times finer, sufficient to resolve the boundary layers that were anticipated. Garaud \& Garaud did reproduce the Brun-Zahn coefficients with an impermeable boundary.) Illustrated are streamlines, magnetic field lines, and greyscale representations of temperature perturbations and the angular velocity in quadrants of the Sun, underneath which are enlargements of the tachocline region $\left(r>0.65 \mathrm{R}_{\odot}\right)$ plotted as functions of latitude and radius, $r$. It is evident that a tachocline has been established, and that, for the first time in a global simulation, the large-scale behaviour of the differential rotation of the convection zone has not been imprinted at great depths in the radiative interior. There is some spatial variation in the interior angular velocity, however, on a relatively small scale which is not evident in Fig. 1. Garaud \& Garaud suggest that the averaging kernels for Fig. 1 are broad enough not to have resolved the structure, and that in any case the features are probably much stronger than is likely to exist in the Sun because it had been necessary to augment the magnitudes of both the imposed flow velocities and the magnetic field in order to produce a magnetic Reynolds number that exceeds unity with the enormously increased diffusivities that it had been necessary to adopt.

In the face of these encouraging beginnings there remain some outstanding issues. The most blatant is the angular velocity $\Omega_{0}$ of the inert core, which is in torque-free equilibrium with the outer part of the radiative zone. Its value is $0.875 \Omega_{\text {eq }}$, whereas seismological analysis of the Sun, as I have already pointed out, yields $0.93 \Omega_{\mathrm{eq}}, \Omega_{\mathrm{eq}}$ being the equatorial angular velocity of the convection zone. The discrepancy is unexplained, although Garaud \& Guervilly (2008) are making some progress towards rectifying that. The discrepancy may be merely a product of not having achieved a low-enough value of the diffusivity augmentation factor $\tilde{f}$.

Another obvious deficiency of the model is that the vertical flow through the base of the convection zone has been imposed artificially. A significant advance would be to couple the tachocline to a simple yet not too unrealistic model of the convection zone, with, say, a representation of the effect of the anisotropic Reynolds stresses on the large-scale flow to generate the gyroscopic pumping. I eagerly await the outcome of such a calculation.

\section{Closing Remarks}

I conclude with three brief remarks concerning alternative views of the overall dynamics. I trust it is evident, even if I have not given it as much emphasis as others might, that the general picture that I have advocated is far from being universally accepted. As I pointed out in the previous section, Brun \& Zahn (2006), for example, believe that the principal model I have discussed lacks 
some further essential ingredient. I shall do little more than simply mention them.

My first point concerns the angular-momentum transport by the layerwise two-dimensional turbulence envisaged by Spiegel \& Zahn (1992) in their tachocline model. As Gough \& McIntyre (1998) pointed out, such motion is not expected to lead to diffusive mixing of angular velocity - indeed it is not even everywhere diffusive of anything - but in places transports angular momentum in a wave-like manner - it is what some people have called antidiffusive, with a tendency to mix potential vorticity (Garaud 2001; see also Gough \& Lynden-Bell 1968). McIntyre (2003) provides a more extensive discussion, citing a critical analysis by Haynes et al. (1991) showing that at least in the rectilinear case straightforward local momentum mixing by turbulence is not possible without accompanying wave transport. However, there is a more recent, and rather different, numerical simulation by Miesch (2003) which appears to lead Miesch to conclude the contrary, and which therefore demands comment. Miesch considered a spherical shell of fluid between impenetrable, isothermal, horizontal, stress-free boundaries, initially rotating uniformly with angular velocity $\Omega_{0}$. He supplied the vertical component of the momentum equation with an axisymmetric torque whose magnitude declined with depth, to mimic forcing from above by differentially rotating overshooting convection. He also added a distribution of random sources of either vertical vorticity, to generate Rossby waves, or horizontal velocity divergence, to generate gravity waves, the locations of those sources rotating rigidly (not moving with the fluid), also with angular velocity $\Omega_{0}$. Not surprisingly, wave drag from dissipation tended to move the differentially forced flow towards the state of uniform rotation assumed by the externally imposed grid of sources, which Miesch interpreted as a tendency for internally generated turbulence to oppose rotational shear. Evidently a similar calculation in which the wave sources are contained by the fluid, the fluid receiving no externally imposed torque other than the differential forcing from above, would be much more informative.

Propagation and subsequent dissipation of downwardly propagating gravity waves generated in the convection zone is also a way of transporting angular momentum through the tachocline, but when such transport was first mooted as a way of modifying the angular velocity of the Sun (Gough 1977) it was considered not to lead to uniform rotation. I have in mind the mechanism now believed to drive the quasibiennial oscillation of the Earth's atmosphere, a mechanism which was dramatically demonstrated by Plumb \& McEwan (1978), and which arises from the phenomenon that prograde gravity waves, transporting positive angular momentum, dissipate more readily than retrograde waves, at a rate which increases with increasing angular velocity of the fluid, thereby enhancing shear. However, this mechanism has not been generally accepted by astrophysicists as being the dominant determinant of gravity-wave angular-momentum transport through the Sun. There are two principal issues: the first is whether or not the waves are generated to an amplitude great 
enough for the transport to be significant, the second is whether they enhance the shear or suppress it. These issues have been reviewed by Gough (2002) and, from a rather different perspective, by Charbonnel \& Talon (2007). I think it is true to say that they have not yet been resolved to everyone's satisfaction. So the debate will continue.

Finally, I comment on a claim by Forgács-Dajka \& Petrovay (2001, 2002; also Petrovav 2003; Forgács-Daika 2004) that an oscillating horizontal magnetic field, presumed to have been generated by a dynamo in the convection zone, and diffusing into the radiative interior of the Sun from above, applies a stress on the fluid which opposes other agents acting on the fluid that tend to transfer latitudinal differential rotation. It is argued that it causes the radiative zone to rotate rigidly, the transition occuring on a lengthscale which, with a suitable choice of conditions, could correspond to the solar tachocline. Even though the early work was criticized at the Solar Tachocline meeting held at the Isaac Newton Institute of Mathematical Sciences, Cambridge in 2004 (Gough 2007), for presuming the field presented to the tachocline to be latitudinally unsheared, and thereby imparting an unsheared Maxwell stress on the radiative zone which would naturally tend to oppose any other tendency to shear, the idea in some circles appears to have been given almost equal credance to the more sophisticated dynamical discussions (e.g., Zahn 2007; Brun \& Zahn 2006). Therefore it behoves anyone wanting to establish the true dynamical balance to look at this model more critically. To this end, Jean-Paul Zahn and I have initiated a simple calculation to assess the effect of the convection-zone shear on the Maxwell stresses, and to estimate the outcome for a Forgács-Dajka and Petrovay model. The idea is to imagine the dynamo field to be generated by a suitable imposed current distribution in the convection zone, and to calculate the resulting magnetic field assuming the convection zone to be rotating as is observed, endowing the fluid in the convection zone with a uniform scalar turbulent magnetic diffusivity. We have not yet reached an agreed conclusion concerning the dynamical response of the nonturbulent fluid beneath. But what we have established is that if the radiative interior were initially rotating uniformly, the stress transported would have been such as to induce latitudinal differential rotation in the same sense as that of the convection zone, as one would expect. Whether the subsequent evolution would induce a latitudinal shear that is intense enough to be tested by seismology remains to be seen.

Acknowledgement. I am very grateful to Paula Younger for typing the manuscript, to Günter Houdek and Jesper Schou for helping with the figures, and to Rob Rutten for conversion to the publisher's format.

\section{References}

Baldner, C. S., Basu, S. 2008, ApJ, 686, 1349 
Basu, S., Antia, H. M. 2003, ApJ, 585, 553

Basu, S., Christensen-Dalsgaard, J., Chaplin, W. J., et al. 1997, MNRAS, 292, 243

Braithwaite, J., Spruit, H. C. 2004, Nat, 431, 819

Brown, T. M., Christensen-Dalsgaard, J., Dziembowski, W. A., et al. 1989, ApJ, 343,526

Brown, T. M., Morrow, C. A. 1987, ApJ, 314, L21

Brun, A. S., Zahn, J.-P. 2006, A\&A, 457, 665

Chaboyer, B., Zahn, J.-P. 1992, A\&A, 253, 173

Charbonneau, P., Dikpati, M., Gilman, P. A. 1999, ApJ, 526, 523

Charbonnel, C., Talon, S. 2005, Science, 309, 2189

Charbonnel, C., Talon, S. 2007, in Unsolved Problems in Stellar Physics: A Conference in Honor of Douglas Gough, eds. R. J. Stancliffe, J. Dewi, G. Houdek, \& C. A. R. G. Martin, Tout, Am. Inst. Phys. Conf. Series, 948, 15

Christensen-Dalsgaard, J., Dappen, W., Ajukov, S. V., et al. 1996, Science, 272, 1286

Christensen-Dalsgaard, J., Gough, D. O., Thompson, M. J. 1991, ApJ, 378, 413

Christensen-Dalsgaard, J., Schou, J. 1988, in Seismology of the Sun and Sun-Like Stars, ed. E. J. Rolfe, ESA-SP, 286, 149

Corbard, T., Blanc-Féraud, L., Berthomieu, G., Provost, J. 1999, A\&A, 344, 696

Duvall, Jr., T. L., Dziembowski, W. A., Goode, P. R., et al. 1984, Nat, 310, 22

Dziembowski, W. A., Goode, P. R. 1989, ApJ, 347, 540

Dziembowski, W. A., Goode, P. R., Libbrecht, K. G. 1989, ApJ, 337, L53

Einstein, A. 1926, Naturwissenschaften, 14, 223

Elliott, J. R., Gough, D. O. 1999, ApJ, 516, 475

Elsworth, Y., Howe, R., Isaak, G. R., et al. 1995, Nat, 376, 669

Forgács-Dajka, E. 2004, A\&A, 413, 1143

Forgács-Dajka, E., Petrovay, K. 2001, Solar Phys., 203, 195

Forgács-Dajka, E., Petrovay, K. 2002, A\&A, 389, 629

Garaud, P. 2001, MNRAS, 324, 68

Garaud, P. 2002, MNRAS, 329, 1

Garaud, P. 2007, in The Solar Tachocline, eds. D. W. Hughes, R. Rosner, \& N. O. Weiss, 147

Garaud, P., Brummell, N. H. 2008, ApJ, 674, 498

Garaud, P., Garaud, J.-D. 2008, MNRAS, 391, 1239

Garaud, P., Guervilly, C. 2008, ArXiv e-print

Gilman, P. A., Howe, R. 2003, in GONG+ 2002. Local and Global Helioseismology: the Present and Future, ed. H. Sawaya-Lacoste, ESA-SP, 517, 283

Gough, D. O. 1977, in The Energy Balance and Hydrodynamics of the Solar Chromosphere and Corona, eds. R. M. Bonnet \& Ph. Delache, IAU Colloq. 36, 3

Gough, D. O. 1984, Adv. Space Res., 4, 85

Gough, D. O. 1985, in Future missions in solar, heliospheric and space plasma physics, eds. E. J. Rolfe \& B. Battrick, ESA-SP, 235, 183

Gough, D. O. 1997, Nat, 388, 324

Gough, D. O. 2002, in From Solar Min to Max: Half a Solar Cycle with SOHO, ed. A. Wilson, ESA-SP, 508, 577

Gough, D. O. 2007, in The Solar Tachocline, eds. D. W. Hughes, R. Rosner, \& N. O. Weiss, 3

Gough, D. O., Lynden-Bell, D. 1968, J. Fluid Mech., 32, 437

Gough, D. O., McIntyre, M. E. 1998, Nat, 394, 755 
Haynes, P. H., McIntyre, M. E., Shepherd, T. G., Marks, C. J., Shine, K. P. 1991, J. Atmos. Sciences, 48, 651

Howe, R., Christensen-Dalsgaard, J., Hill, F., et al. 2000, Science, 287, 2456

Kosovichev, A. G. 1996, ApJ, 469, L61

Kumar, P., Quataert, E. J. 1997, ApJ, 475, L143

MacGregor, K. B., Charbonneau, P. 1999, ApJ, 519, 911

Markey, P., Tayler, R. J. 1973, MNRAS, 163, 77

McIntyre, M. E. 2003, in Stellar astrophysical fluid dynamics, eds. M. J. Thompson, J. Christensen-Dalsgaard, Cambridge University Press, 111

Miesch, M. S. 2003, ApJ, 586, 663

Petrovay, K. 2003, Solar Phys., 215, 17

Plumb, R. A., McEwan, A. D. 1978, J. Atmos. Sciences, 35, 1827

Rüdiger, G., Kitchatinov, L. L. 1997, Astron. Nachrichten, 318, 273

Schou, J., Antia, H. M., Basu, S., et al. 1998, ApJ, 505, 390

Spiegel, E. A. 1972, NASA Special Publication, 300, 61

Spiegel, E. A., Zahn, J.-P. 1992, A\&A, 265, 106

Wood, T. S., McIntyre, M. E. 2007, in Unsolved Problems in Stellar Physics: A Conference in Honor of Douglas Gough, eds. R. J. Stancliffe, J. Dewi, G. Houdek, \& C. A. R. G. Martin, Tout, Am. Inst. Phys. Conf. Series, 948, 303

Zahn, J.-P. 2007, in The Solar Tachocline, eds. D. W. Hughes, R. Rosner, \& N. O. Weiss, 89

Zahn, J.-P., Talon, S., Matias, J. 1997, A\&A, 322, 320 